\title{
Avaliação de sistemas de irrigação por gotejamento, utilizados na cafeicultura ${ }^{1}$
}

\author{
Luís O. C. de Souza ${ }^{2}$, Everardo C. Mantovani ${ }^{2}$, Antonio A. Soares ${ }^{2}$, Márcio M. Ramos ${ }^{2} \&$ Paulo S. L. de Freitas ${ }^{3}$
}

\begin{abstract}
RESUMO
Este trabalho foi conduzido no período de agosto a outubro de 1999, com o objetivo de avaliar os sistemas de irrigação por gotejamento utilizados na cafeicultura irrigada do norte do Espírito Santo e nas áreas de cerrado de Minas Gerais e caracterizar a porcentagem de área molhada (PAM) nesses sistemas de irrigação. Foram realizadas medidas das dimensões do bulbo molhado para determinação da porcentagem de área molhada. Pelos resultados obtidos verificou-se que, dos valores de coeficiente de uniformidade de distribuição determinados, apenas um sistema apresentou valor abaixo de $70 \%$, refletindo uma uniformidade ruim. No que se refere aos demais resultados, dez sistemas indicaram valores de uniformidade de distribuição entre 70 e $80 \%$, oito sistemas mostraram valores entre 80 e $90 \%$ e doze sistemas apontaram valores acima de 90\%, indicando uniformidade razoável, boa e excelente, respectivamente. Dos valores determinados, a maioria dos sistemas que apresentaram uniformidade de boa a excelente estava situada na região de cerrado de Minas Gerais. Com relação à PAM, os valores determinados variaram na faixa de 19 a 100\% e apenas um sistema apresentou valor abaixo do limite mínimo recomendado para regiões úmidas, que é de $20 \%$.
\end{abstract}

Palavras-chave: uniformidade de aplicação, avaliação da irrigação, porcentagem de área molhada

\section{Evaluation of trickle irrigation systems used in coffee crop}

\begin{abstract}
This study was carried out over the period from August to October 1999, aiming the evaluation of trickle irrigation systems used in coffee crop in the northern part of Espírito Santo State and the savannah areas of Minas Gerais State, as well as to characterize the percentage of wetted area (PWA) of these irrigation systems. The wetted bulb dimensions measurements were accomplished for determining the percent wetted area. According to the results, just one system presented a value lower than $70 \%$ for the distribution uniformity coefficient, reflecting a bad uniformity. Concerning to the other results, ten systems showed the distribution uniformity values ranging from 70 to $80 \%$, whereas for eight systems, it ranged from 80 to $90 \%$, and for twelve systems the uniformity was above $90 \%$, which could be considered as reasonable, good and excellent uniformities, respectively. Among the determined values, it was observed that most of the systems presenting good to excellent uniformity were located in the region of Minas Gerais State. In relation to PWA, the values ranged from 19 to $100 \%$, and just one system presented a value below the minimum limit $(20 \%)$ recommended for wetted area.
\end{abstract}

Key words: application uniformity, irrigation evaluation, percentage of wetted area

1 Parte da Dissertação de Mestrado do primeiro autor

${ }^{2}$ DEA/UFV. Av. P.H. Rolfs s/n, CEP 36571-000, Vicosa, MG. Fone: (31) 3899-2734. E-mail: locs_souza@yahoo.com.br; everardo@ufv.br; aasoares@ufv.br; mmramos@ufv.br

${ }^{3}$ DAG/UEM. Av. Colombo 5790, CEP 87020-900, Maringá, PR. Fone: (44) 3261-4316. E-mail: pslfreitas@uem.br 


\section{INTRODUÇÃO}

Vários sistemas de irrigação podem ser utilizados na cafeicultura, como gotejamento, microaspersão, mangueiras perfuradas, aspersão convencional, pivô central e autopropelido. A escolha do sistema mais adequado depende de vários fatores, podendo-se destacar a topografia e o tipo de solo, a fonte de água (localização, vazão e qualidade), o sistema de plantio do cafezal e o custo do equipamento e de sua operação.

Dentre os sistemas de irrigação utilizados na cafeicultura, a irrigação por gotejamento tem-se destacado principalmente pela alta uniformidade de aplicação de água alcançando, assim, alta eficiência de aplicação. Essas características de desempenho propiciam outra vantagem da utilização da irrigação por gotejamento ou seja, a aplicação de produtos químicos via água de irrigação (quimigação).

Para se avaliar em que condições o sistema de irrigação está operando, parâmetros de desempenho devem ser definidas com base em determinações de campo, como vazão, uniformidade de aplicação e tempo de irrigação.

Um dos principais parâmetros usados na avaliação de um sistema de irrigação é a uniformidade de aplicação de água sobre a área irrigada. Uma baixa uniformidade de distribuição de água reduz a eficiência de aplicação de água e a produtividade.

A eficiência de irrigação é um conceito largamente utilizado, tanto em projetos quanto no manejo de sistemas de irrigação. Segundo Keller \& Bliesner (1990), o conceito de eficiência pode ser dividido em dois aspectos básicos: em uniformidade de aplicação e nas perdas que podem ocorrer durante a operação do sistema.

Sammis \& Wu (1985) sugerem que uma manutenção imprópria do sistema de irrigação pode causar decréscimo de $60 \%$ ou mais na uniformidade de aplicação, resultando em aumento na quantidade de água aplicada, para compensar a baixa uniformidade e a redução na produtividade

Com relação à qualidade dos gotejadores, um coeficiente de variação de fabricação baixo, a variação das características do gotejador devido à temperatura, problemas com entupimento e tempo de uso, são os fatores que mais afetam a uniformidade de um sistema de irrigação por gotejamento (Solomon, 1985; Pizarro Cabello, 1990; López et al., 1992).

Bonomo (1999), avaliando sistemas de irrigação empregados na cafeicultura em regiões de cerrado de Minas Ge- rais, encontrou baixos valores de coeficiente de uniformidade de distribuição de água, considerados inadequados para sistemas de irrigação por gotejamento (Merriam \& Keller, 1978). Esses valores não refletiram, possivelmente, a real situação, havendo necessidade de uma avaliação mais ampla, sobretudo com relação ao número de sistemas avaliados e fabricantes, enfatizando também a idade do equipamento.

Com o aumento da demanda de sistemas de irrigação por parte das lavouras cafeeiras ressalta a olhos vistos, a necessidade de maiores pesquisas na área, com o intuito de determinar o desempenho dos equipamentos utilizados e o nível do manejo de irrigação para esta cultura.

Considerando-se o exposto, o objetivo do presente trabalho foi avaliar, de acordo com os parâmetros de engenharia, a uniformidade de distribuição de água dos sistemas de irrigação por gotejamento, correlacionando-os com as características do projeto e de operação do sistema, e caracterizar a atual situação da porcentagem de área molhada desses sistemas de irrigação, empregados na cafeicultura irrigada, nas regiões produtoras do norte do Espírito Santo e em áreas de cerrado de Minas Gerais.

\section{MATERIAL E MÉTODOS}

\section{Localização e caracterização dos sistemas avaliados}

O trabalho de campo foi realizado no período de agosto a outubro de 1999, em 31 sistemas de irrigação por gotejamento, situados nas regiões cafeicultoras do norte do Espírito Santo e em áreas de cerrado de Minas Gerais, que englobam as regiões do Triângulo Mineiro e Alto Paranaíba. Devido às características fisiológicas das espécies de café e das aptidões climáticas das regiões estudadas no norte do Espírito Santo, foram avaliadas propriedades produtoras de Coffea canephora cv. Conilon e, nas áreas de cerrado de Minas Gerais, propriedades produtoras de Coffea arabica. A determinação das propriedades avaliadas se fez com base em informações coletadas nas cooperativas, associações de cafeicultores e secretarias de agricultura existentes nos municípios em estudo.

Dos sistemas avaliados na região norte do Espírito Santo, quatro são classificados como alternativos, denominados, na região, de espirro ou espirramento, também chamados tubo de geladeira (Figura 1A) ou gravatinha (Figura 1C); o primeiro recebe este nome por ser confeccionado com um
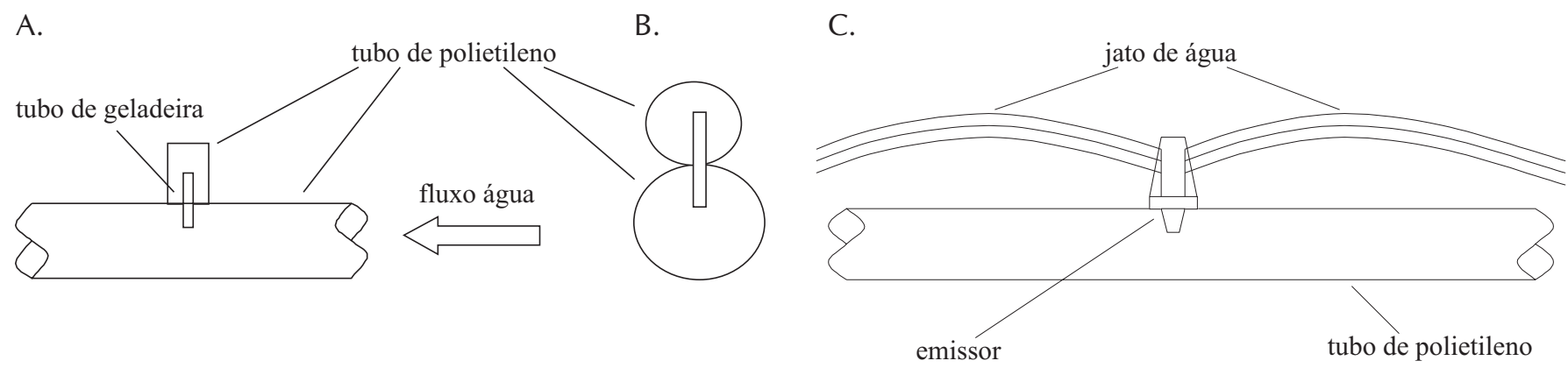

Figura 1. Esquema ilustrativo do sistema alternativo tubo de geladeira. (A) corte longitudinal; (B) corte transversal e (C) do sistema alternativo gravatinha 
pedaço de tubo de cobre empregado no sistema de refrigeração de geladeira, de aproximadamente $2,0 \mathrm{~cm}$ de comprimento, inserido em um tubo de polietileno, que serve como linha lateral, possuindo um anteparo feito de parte deste tubo de polietileno, enquanto o segundo recebe este nome devido à forma como a água é emitida através do emissor, ao longo da linha lateral, assemelhando-se a uma gravata borboleta. Para o dimensionamento desses dois sistemas alternativos é colocado um emissor por planta.

\section{Uniformidade de aplicação de água}

A uniformidade de distribuição de água foi determinada utilizando-se a metodologia proposta por Keller \& Karmeli (1975), modificada por Denículi et al. (1980), que consiste na coleta da vazão dos gotejadores em oito pontos ao longo da linha lateral e em quatro linhas laterais, ao longo da linha de derivação. As linhas laterais selecionadas foram aquelas situadas no início da linha de derivação, a 1/3 da origem da linha de derivação, a $2 / 3$ da origem da linha de derivação e no final desta.

Os oito pontos selecionados em cada linha lateral foram os situados no início da linha lateral, a 1/7, 2/7, 3/7, 4/7, 5/ 7 e a $6 / 7$ do comprimento da linha lateral, e aquele situado no final. Coletou-se o volume que cada planta recebia, enquanto o intervalo de tempo utilizado para determinação da vazão foi de 5 min.

As medidas de pressão, utilizando-se um manômetro de Bourdon graduado de 0 a 6 bar, foram feitas apenas nos finais das linhas laterais, devido à impossibilidade encontrada em campo de se acoplá-lo no início das linhas laterais, pois as linhas de derivação se encontravam enterradas. Em apenas dois sistemas avaliados foi possível o acoplamento do manômetro para a medida da pressão no início da linha lateral, e em outro foi possível acoplar o manômetro no cabeçote da linha de derivação.

O coeficiente de uniformidade de distribuição (CUD) foi definido utilizando-se a Eq. 1, apresentada por Keller \& Karmeli (1975). Para determinação da uniformidade estatística $\left(U_{\mathrm{s}}\right)$ lançou-se mão da Eq. 2, e os respectivos intervalos de confiança foram determinados de acordo com metodologia proposta por Bralts \& Kesner (1983) que utilizam a combinação das Eqs. 3 e 4, em um nível de significância de 5\%.

$$
\mathrm{CUD}=100 \frac{\mathrm{q}_{25}}{\overline{\mathrm{q}}}
$$

em que $\mathrm{q}_{25 \%}$ é a média de $25 \%$ dos menores valores de vazões observadas.

$$
\mathrm{U}_{\mathrm{s}}=100(1-\mathrm{CV})
$$

em que:

$\mathrm{U}_{\mathrm{s}}$ - uniformidade estatística, \%

CV - coeficiente de variação de vazão dos gotejadores

$$
\mathrm{P}\left(\mathrm{CV}-\mathrm{t}\left(\frac{\alpha}{2}\right) \mathrm{S}_{\mathrm{CV}} \leq \mathrm{CV} \leq \mathrm{CV}+\mathrm{t}\left(\frac{\alpha}{2}\right)^{\left.\mathrm{S}_{\mathrm{CV}}\right)=1-\alpha}\right.
$$

em que:

$$
\begin{aligned}
\mathrm{CV} & - \text { coeficiente de variação } \\
\text { to } & - \text { valor de } \mathrm{t} \text { student para dado } \\
\alpha & - \text { nível de significância desejado } \\
\mathrm{S}_{\mathrm{CV}} & \text { desvio-padrão do coeficiente de variação, cal- } \\
& \text { culado pela equação: }
\end{aligned}
$$

$$
\mathrm{S}_{\mathrm{CV}}=\frac{\mathrm{CV}}{\sqrt{2 \mathrm{n}}} \sqrt{1+2\left(\mathrm{CV}^{2}\right)}
$$

em que n é o número de observações.

\section{Porcentagem de área molhada}

Na determinação da área molhada utilizou-se a metodologia proposta por Merriam \& Keller (1978), que consiste na determinação das medidas do bulbo molhado formado pelo emissor, depois de concluído o tempo total de irrigação, em pontos predeterminados na área, próximos às plantas onde foram coletados os volumes para determinação da vazão. Esses pontos foram: no início da primeira linha lateral; a $1 / 3$ do comprimento da linha lateral localizada a $1 / 3$ do comprimento da linha de derivação; a $2 / 3$ do comprimento da linha lateral localizada a $2 / 3$ do comprimento da linha de derivação e a 3/3 do comprimento da última linha lateral.

Foram cavadas pequenas trincheiras ao redor da planta, com auxílio de enxada, para melhor se estimar a real extensão do bulbo formado, visto que a maior área horizontal do bulbo não ocorre na superfície e, sim, a alguns centímetros de profundidade. A porcentagem de área molhada foi estimada dividindo-se a área do bulbo molhado formado pelo emissor pelo espaçamento entre emissores e as linhas laterais.

\section{RESULTADOS E DISCUSSÃO}

\section{Uniformidade de aplicação de água dos sistemas avaliados na região norte do Espírito Santo}

A Tabela 1 apresenta os valores do coeficiente de uniformidade de distribuição (CUD) e uniformidade estatística $\left(\mathrm{U}_{\mathrm{s}}\right)$ determinados para os sistemas de irrigação por gotejamento, avaliados na região norte do Espírito Santo. Os valores de uniformidade estatística vêm seguidos dos respectivos valores de intervalo de confiança.

Nota-se que, dos 16 sistemas avaliados na região norte do Espírito Santo, apenas um apresentou valor de CUD abaixo de $70 \%$ classificado, portanto, como ruim, pelo critério proposto por Merriam \& Keller (1978). Neste sistema já se observava, visualmente, uma baixa uniformidade pois, no intuito de resolver problemas freqüentes de entupimento, causados pela alta concentração de ferro na água de irrigação, o proprietário retirou, de alguns gotejadores, a membrana de borracha que atuava como mecanismo de autocompensação do gotejador, provocando grande variação de vazão ao longo da linha lateral, o que pode ser visualizado na Figura 2A, na qual é apresentada a vazão dos emissores ao longo das linhas laterais (sistema 1). 
Tabela 1. Coeficiente de uniformidade de distribuição (CUD), uniformidade estatística $\left(U_{s}\right)$ e intervalo de confiança $(I C)$, para os sistemas de gotejamento avaliados na região norte do Espírito Santo

\begin{tabular}{rlccc}
\hline Sistema & \multicolumn{1}{c}{ Tipo } & CUD (\%) & U $_{\mathbf{s}}(\%)$ & IC (\%) \\
\hline 1 & autocompensante & 48 & 44 & 18,4 \\
\hline 2 & alternativo & 70 & 75 & 6,8 \\
3 & alternativo & 73 & 78 & 6,8 \\
4 & alternativo & 73 & 76 & 6,6 \\
\hline 5 & alternativo & 75 & 82 & 4,7 \\
6 & autocompensante & 76 & 81 & 5,1 \\
\hline 7 & tubogotejador & 76 & 80 & 5,3 \\
8 & tubogotejador & 77 & 84 & 4,1 \\
\hline 9 & tubogotejador & 77 & 81 & 5,0 \\
10 & autocompensante & 86 & 87 & 3,8 \\
\hline 11 & autocompensante & 86 & 89 & 2,8 \\
12 & autocompensante & 88 & 88 & 3,2 \\
13 & tubogotejador & 88 & 90 & 2,6 \\
\hline 14 & tubogotejador & 90 & 91 & 2,2 \\
15 & autocompensante & 90 & 92 & 2,1 \\
16 & autocompensante & 96 & 97 & 0,7 \\
\hline
\end{tabular}

Dos sistemas restantes, oito apresentaram CUD entre 70 e $80 \%$, quatro mostraram valores entre 80 e $90 \%$ e apenas três resultaram em valores acima de $90 \%$, podendo ser classificados como razoáveis, bons e excelentes, respectivamente (Merriam \& Keller, 1978).

Dos sistemas classificados como razoáveis, quatro são denominados alternativos, ou seja, utilizam emissores construídos sem qualquer especificação técnica; em três deles (sistemas 2, 3 e 4), os problemas foram causados principalmente pelo inadequado dimensionamento hidráulico da linha lateral, cujo comprimento excessivo (para o emissor considerado), ou mesmo uma altura manométrica insuficiente (para o projeto), implicou em uma variação de vazão acima do recomendado para sistemas de irrigação localizada. Lopéz et al. (1992) definiram que esta variação de pressão ao longo da linha lateral não deve proporcionar variação de vazão superior a $10 \%$.

No sistema alternativo restante (sistema 5), apesar de um estudo hidráulico adequado para dimensionamento da linha lateral, as limitações na fabricação do emissor, que não apre-

A.

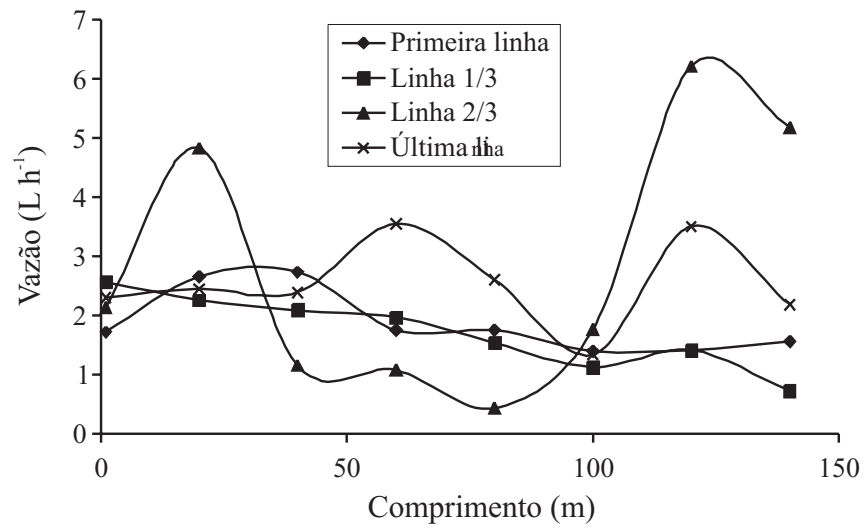

sentava adequada padronização do diâmetro interno, proporcionaram valor inadequado de uniformidade, devido a variações de vazão ao longo da linha lateral. Na Figura 2B apresenta-se a vazão dos emissores ao longo das linhas laterais para um destes sistemas alternativos (sistema 2), observando-se variação de vazão ao longo da linha lateral superior ao limite máximo recomendado, com valores médios acima de $50 \%$.

Os quatro sistemas (Tabela 1) com valores de uniformidade classificados como razoáveis, na faixa de 70 a $80 \%$ (sistemas 6, 7, 8 e 9), apresentaram comprimento da linha lateral superior ao recomendado pelos critérios do fabricante do equipamento.

Esses valores, relacionados ao dimensionamento das linhas laterais ocasionam, no final, uma pressão de serviço inferior à indicada pelo fabricante, o que causa variação de vazão ao longo da linha lateral superior ao valor máximo de $10 \%$ recomendado; contribuiu também para a obtenção de valores baixos de uniformidade, o fato de três sistemas (sistemas 6, 7 e 8) apresentarem grande número de gotejadores entupidos e com o final das linhas laterais cheias de ar; no entanto, a uniformidade de distribuição desses sistemas pode ser melhorada por meio de práticas de operação do equipamento mais criteriosas, como melhoria no sistema de filtragem, limpeza ou substituição dos gotejadores entupidos e limpeza das linhas laterais.

Ainda segundo critério apresentado por Merriam \& Keller (1978), quatro sistemas (sistemas 10, 11, 12 e 13) apresentaram CUD entre 80 e $90 \%$, classificados como bons, e três sistemas (sistemas 14, 15 e 16) apontaram CUD acima de $90 \%$, sendo classificados como excelentes (Tabela 1). Esses valores de uniformidade refletem o bom dimensionamento hidráulico, pois as pressões de serviço no final das linhas laterais se encontravam dentro dos limites de operação recomendados pelos fabricantes; além disso, as condições de operação desses sistemas, onde são feitas limpezas periódicas dos gotejadores e das linhas laterais, e a reposição dos gotejadores entupidos ou danificados, também contribuíram para obtenção desses valores de uniformidade.

Ressaltando-se a importância da obtenção de altas uniformidades de aplicação de água em sistemas localizados,

B.

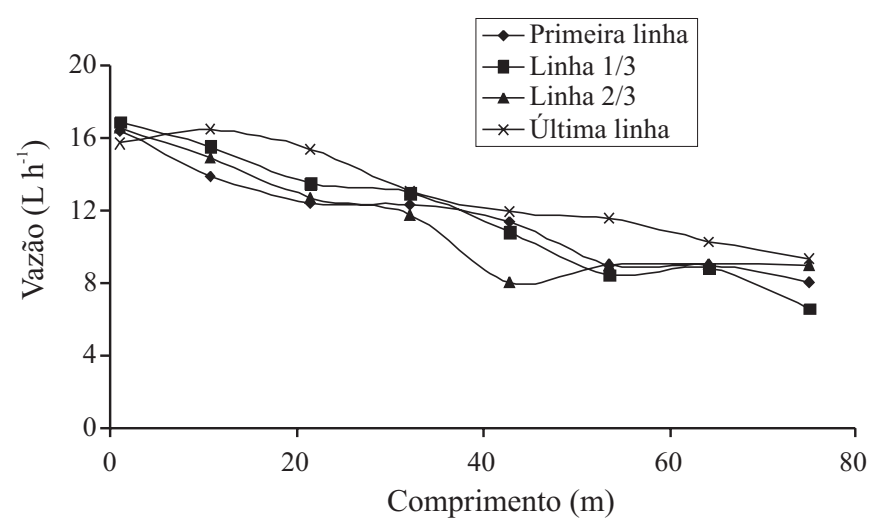

Figura 2. Vazão dos emissores ao longo das linhas laterais avaliadas, sistema 1, região norte do ES (CUD = 48\%) (A) e (B) sistema 2, região norte do ES $(\mathrm{CUD}=70 \%$ ) 
A.

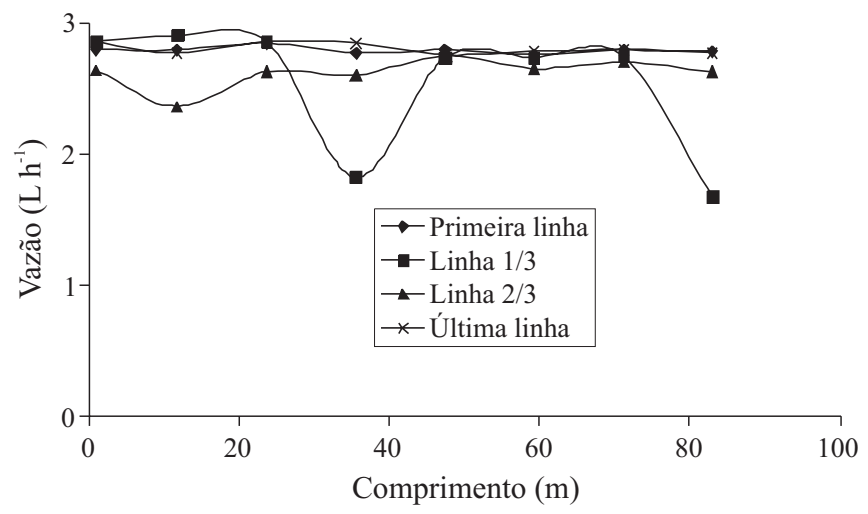

B.

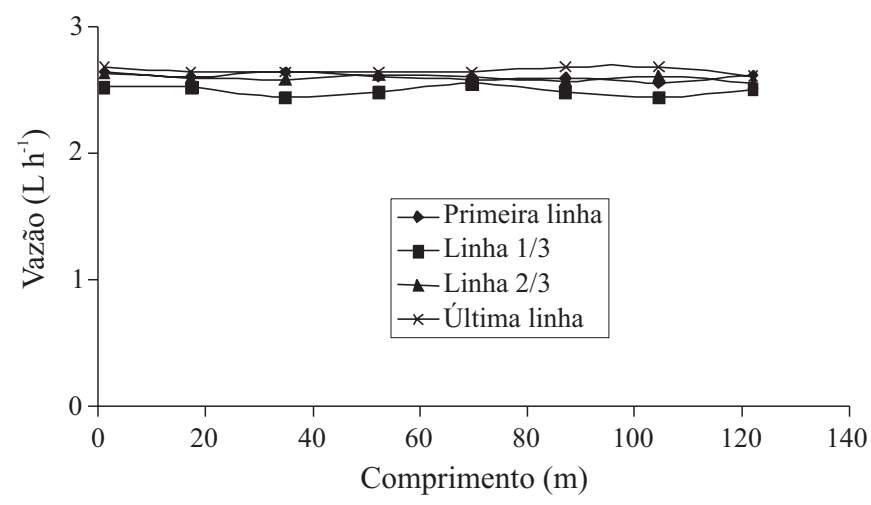

Figura 3. Vazão dos emissores ao longo das linhas laterais avaliadas, sistema 13 , região norte do ES (CUD $=88 \%$ ) (A) e sistena 16 , região norte do ES $(\mathrm{CUD}=96 \%)(\mathrm{B})$

conclui-se que os coeficientes adotados, principalmente o CUD, são sensíveis a pequenas variações na distribuição de água de um sistema, o que pode ser explicado comparando-se as Figuras 3A e 3B. Observa-se que a diferença básica no sistema classificado como excelente (Figura 3B), em relação ao sistema classificado como bom (Figura 3A), se situa basicamente em três variações na vazão dos gotejadores deste sistema, duas ocorridas na linha a $1 / 3$ da origem e outra na linha, a $2 / 3$ da origem, Motivo pelo qual o sistema representado na Figura $3 \mathrm{~A}$ obteve uniformidade menor que aquela determinada para o sistema representado pela Figura $3 \mathrm{~B}$, sugerindo que a metodologia utilizada pode estar sujeita a erros, uma vez que trabalha com pequeno número de linhas laterais, como amostragem.

Apresentam-se, na Tabela 2, as variações de vazão verificadas nas linhas laterais e nos setores avaliados. Observase que os valores de variação de vazão foram inaceitáveis para sistemas de irrigação por gotejamento; o único que apresentou valor adequado de variação de vazão, recomendado por
López et al. (1992), foi o sistema 16, que valor reflete o coeficiente de uniformidade determinado para este sistema, classificado como excelente (Merriam \& Keller, 1978); no entanto, os demais sistemas indicam variações de vazão muito acima do limite recomendado, inclusive os dois sistemas restantes, que apresentaram valores de coeficiente de uniformidade classificados também como excelentes (acima de $90 \%$ ), sugerindo que a metodologia empregada para determinação da uniformidade apresenta falhas, quando aplicada na determinação da variação de vazão, por trabalhar com valores pontuais de coleta de vazão dos gotejadores.

Com relação à uniformidade estatística (Us) vê-se, na Tabela 1, que um sistema (sistema 1) apresentou valor inaceitável (menor que 60\%), três (sistemas 2, 3 e 4) mostraram valores classificados como razoáveis (entre 70 e $80 \%$ ), oito (sistemas 5, 6, 7, 8, 9, 10, 11 e 12) apresentaram valores muito bons (entre 80 e $90 \%$ ) e apenas quatro (sistemas $13,14,15$ e 6 ) os apresentaram como excelentes (acima de $90 \%$ ), de acordo com classificação proposta por Bralts \&

Tabela 2. Variação da vazão ao longo das linhas laterais avaliadas e no setor de cada sistema

\begin{tabular}{|c|c|c|c|c|c|c|c|c|c|c|c|}
\hline \multicolumn{6}{|c|}{ Norte do Espírito Santo } & \multicolumn{6}{|c|}{ Cerrado de Minas Gerais } \\
\hline \multicolumn{6}{|c|}{ Variação da vazão (\%) } & \multicolumn{6}{|c|}{ Variação da vazão (\%) } \\
\hline \multirow{2}{*}{ Sistema } & \multicolumn{4}{|c|}{ Linha Lateral } & \multirow{2}{*}{ Setor } & \multirow{2}{*}{ Sistema } & \multicolumn{4}{|c|}{ Linha Lateral } & \multirow{2}{*}{ Setor } \\
\hline & Primeira & $1 / 3$ & $2 / 3$ & Última & & & Primeira & $1 / 3$ & $2 / 3$ & Última & \\
\hline 1 & 60 & 82 & 258 & 98 & 258 & 1 & 33 & 55 & 45 & 72 & 97 \\
\hline 2 & 70 & 86 & 72 & 60 & 86 & 2 & 60 & 78 & 30 & 71 & 105 \\
\hline 3 & 19 & 30 & 59 & 67 & 88 & 3 & 30 & 20 & 49 & 23 & 84 \\
\hline 4 & 74 & 47 & 54 & 44 & 93 & 4 & 25 & 28 & 46 & 36 & 55 \\
\hline 5 & 42 & 50 & 37 & 60 & 60 & 5 & 27 & 32 & 37 & 32 & 42 \\
\hline 6 & 46 & 49 & 71 & 39 & 87 & 6 & 16 & 24 & 17 & 9 & 28 \\
\hline 7 & 32 & 93 & 47 & 74 & 110 & 7 & 10 & 7 & 22 & 26 & 34 \\
\hline 8 & 39 & 39 & 44 & 62 & 62 & 8 & 34 & 8 & 18 & 12 & 34 \\
\hline 9 & 66 & 54 & 8 & 7 & 66 & 9 & 14 & 25 & 17 & 22 & 32 \\
\hline 10 & 37 & 42 & 25 & 35 & 32 & 10 & 9 & 11 & 15 & 11 & 24 \\
\hline 11 & 29 & 32 & 51 & 23 & 55 & 11 & 18 & 10 & 21 & 11 & 25 \\
\hline 12 & 64 & 24 & 11 & 43 & 68 & 12 & 19 & 15 & 7 & 5 & 19 \\
\hline 13 & 3 & 45 & 14 & 4 & 45 & 13 & 6 & 5 & 5 & 8 & 10 \\
\hline 14 & 11 & 44 & 12 & 22 & 47 & 14 & 11 & 3 & 9 & 12 & 14 \\
\hline 15 & 16 & 9 & 9 & 23 & 40 & 15 & 6 & 2 & 2 & 6 & 6 \\
\hline 16 & 3 & 4 & 3 & 3 & 9 & 16 & 33 & 55 & 45 & 72 & 97 \\
\hline
\end{tabular}


Kesner (1983). Os mesmos autores afirmam que, se o número de pontos de amostragem dobrar, os intervalos de confiança decrescerão aproximadamente um terço, em um nível de significância de 5\%.

\section{Uniformidade de aplicação de água dos sistemas avaliados na região de cerrado, de Minas Gerais}

Na Tabela 3 estão apresentados os valores do coeficiente de uniformidade de distribuição (CUD) e uniformidade estatística (Us), para os sistemas de irrigação por gotejamento, avaliados na região de cerrado de Minas Gerais. Os valores de uniformidade estatística vêm seguidos dos respectivos valores de intervalo de confiança.

Tabela 3. Coeficiente de uniformidade de distribuição (CUD), uniformidade estatística $\left(U_{s}\right)$ e intervalo de confiança $(I C)$, para os sistemas de gotejamento avaliados na região de cerrado de Minas Gerais

\begin{tabular}{clccc}
\hline Sistema & \multicolumn{1}{c}{ Tipo } & CUD (\%) & U $_{\text {s }}(\%)$ & IC (\%) \\
\hline 1 & autocompensante & 77 & 81 & 5,0 \\
2 & autocompensante & 78 & 78 & 6,0 \\
3 & autocompensante & 81 & 84 & 4,2 \\
4 & autocompensante & 85 & 88 & 3,1 \\
5 & autocompensante & 87 & 88 & 3,2 \\
6 & autocompensante & 89 & 92 & 2,0 \\
\hline 7 & autocompensante & 91 & 93 & 1,8 \\
8 & autocompensante & 92 & 92 & 2,0 \\
\hline 9 & autocompensante & 92 & 93 & 1,8 \\
10 & autocompensante & 92 & 94 & 1,5 \\
11 & autocompensante & 93 & 95 & 1,4 \\
12 & tubogotejador & 94 & 96 & 1,1 \\
13 & tubogotejador & 96 & 97 & 0,7 \\
14 & tubogotejador & 97 & 98 & 0,5 \\
15 & tubogotejador & 97 & 96 & 1,0 \\
\hline
\end{tabular}

Nota-se que todos os sistemas apresentaram CUD superior a $77 \%$ e, segundo critério apresentado por Merriam \& Keller (1978), dois podem ser considerados razoáveis, quatro bons e nove podem ser acatados como excelentes.

Os sistemas 1 e 2 apresentaram uniformidade classificada como razoável, o que não é adequado para sistemas de irrigação localizada. Esses valores já eram esperados devido

A.

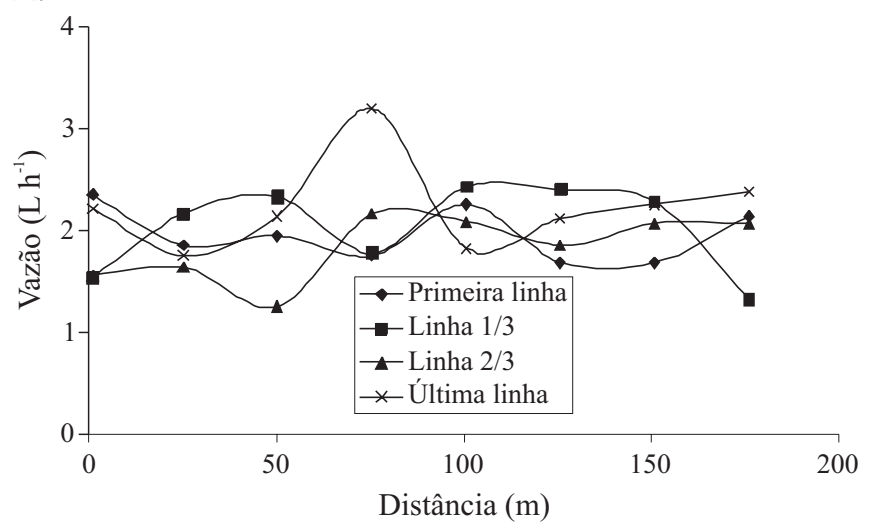

à constatação, durante a avaliação, da presença de gotejadores entupidos, com a membrana de borracha do mecanismo de autocompensação dos gotejadores rompida e até mesmo da falta de alguns gotejadores ao longo da linha lateral. Também se notou presença de bolhas de ar e depósitos minerais e orgânicos no final das linhas laterais; além disso, para esses dois sistemas a variação da vazão ao longo das linhas laterais avaliadas foi muito superior ao limite máximo recomendado de $10 \%$, indicando o motivo da baixa uniformidade de aplicação de água.

Observa-se, na Tabela 2, as variações de vazão ocorridas nas linhas laterais, linhas de derivação e nos setores avaliados, determinados pela Eq. 1.

Ressalta-se que, na maioria dos sistemas, a variação da vazão extrapolou o limite de $10 \%$, mesmo para os sistemas com valores de uniformidade classificados de bom a excelente (CUD $>80 \%$ ); o fato confirma o que foi discutido anteriormente sobre a limitação da metodologia empregada na caracterização da uniformidade e a variação de vazão.

Apresenta-se, na Figura 4A, a vazão dos emissores ao longo da linha lateral, em um sistema avaliado, cujo CUD foi classificado razoável (sistema 1). Verifica-se, também, que existe variação média da vazão dos gotejadores ao longo das linhas laterais, acima de 50\% (Tabela 2).

Os resultados dos sistemas que apresentaram coeficientes de uniformidade de distribuição, classificados como de bom a excelente, estão relacionados a diversos fatores, destacando-se a idade dos sistemas (menos de dois anos de instalação), projetos bem dimensionados, que operam com pressões de serviço dentro da faixa recomendada pelos fabricantes e, ainda, por se tratar de sistemas adequadamente operados em campo, cujos gotejadores e linhas laterais são mantidos limpos e em bom estado de conservação fazendo-se, quando necessário, a substituição dos gotejadores entupidos ou defeituosos. Bonomo (1999), estudando alguns sistemas de irrigação pressurizados utilizados na cafeicultura irrigada em áreas de cerrado de Minas Gerais, encontrou valores de CUD inferiores a $70 \%$ para sistemas de irrigação por gotejamento, valores esses considerados ruins pelos critérios apresentados por Merriam \& Keller (1978). Segundo o autor, referidos valores baixos estão associados ao elevado número de gotejadores entupidos e à presença de bolsas de ar na parte

B.

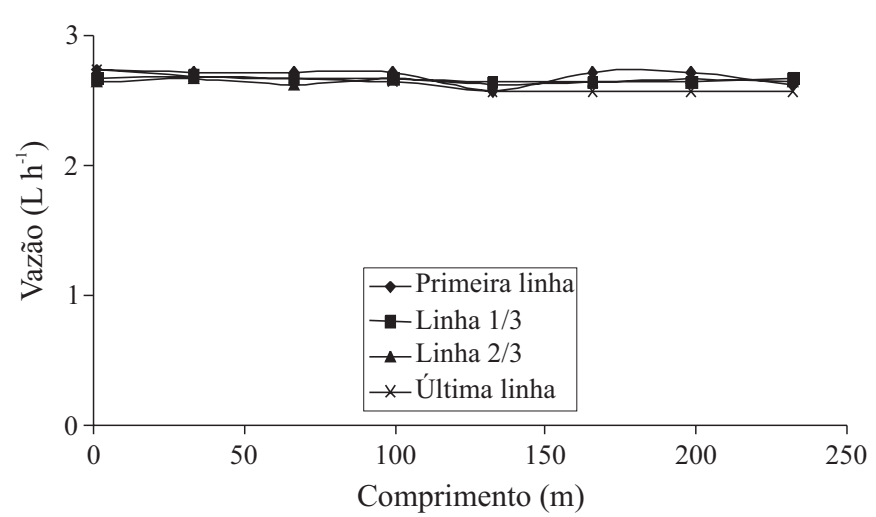

Figura 4. Vazão dos emissores ao longo das linhas laterais avaliadas, sistema 1 (A) (CUD = 77\%) e sistema 15 (B) (CUD = 97\%), na região de cerrado de MG 
final das linhas laterais. Este autor ressalta, também, o fato dos sistemas avaliados por ele serem antigos, refletindo principalmente resultados de sistemas pioneiros e que não apresentam todo o potencial dos atuais sistemas instalados.

$\mathrm{Na}$ Figura 4B tem-se o resultado da avaliação para um sistema no qual o coeficiente de uniformidade foi classificado excelente (sistema 15), de acordo com classificação sugerida por Merriam \& Keller (1978). Observa-se a pequena variação de vazão dos emissores, cujo valor máximo foi de 6\% (Tabela 2).

A uniformidade estatística $\left(\mathrm{U}_{\mathrm{s}}\right)$, de acordo com classificação proposta por Bralts \& Kesner (1983), apresentada na Tabela 3 , mostra que apenas o sistema 2 apresentou valor entre 70 e $80 \%$, sendo classificado como razoável; quatro sistemas $(1,3,4$ e 5$)$ apresentaram valores "muito bom" (entre 80 e $90 \%$ ) e nove sistemas (6 a 15) valores excelentes (acima de 90\%). De acordo com os mesmos autores, os intervalos de confiança determinados (Tabela 3) também se encontram dentro dos limites estabelecidos, como já discutido.

\section{Uniformidade de aplicação de água em função do tempo de uso e do tipo de gotejador}

Um aspecto importante, do ponto de vista de operação de sistemas de irrigação em campo é a relação entre a uniformidade de distribuição de água do sistema com o tempo de implantação ou seu uso.

Na Figura 5A estão apresentadas, para 26 avaliações realizadas no norte do Espírito Santo e no cerrado de Minas Gerais, a distribuição do número de sistemas, em termos de porcentagem, em relação ao tempo de uso, e as faixas de valores de CUD recomendadas por Merriam \& Keller (1978). Nesta análise não se incluíram os sistemas número 1 e os alternativos, avaliados no norte do Espírito Santo; o primeiro, por haver sofrido modificações que comprometeram suas características hidráulicas, e os restantes, por não apresentarem especificações técnicas, capazes de caracterizá-los quanto à vazão nominal, à pressão de serviço e ao dimensionamento hidráulico adequado.

Apesar dos sistemas avaliados estarem em operação por período inferior a três anos, observa-se tendência de sistemas mais velhos (entre dois e três anos) apresentarem CUD menor que os sistemas mais novos (menos de dois anos de uso). Esses resultados indicam problemas relacionados à condução e à operação do equipamento, com o passar do tempo, problemas no sistema de filtragem, reposição de emissores, características da água de irrigação e com projetos inadequados. Andrade Júnior et al. (1997), operando em um sistema com tempo de uso de $3.600 \mathrm{~h}$, mostraram que não houve influência na uniformidade de distribuição deste de um ano para o outro, provavelmente pelo fato do segmento apresentar condições adequadas de operação, condução e manutenção.

Na Figura 5B observar-se a distribuição dos sistemas, de acordo com o tipo de emissor e os valores dos coeficientes determinados. Além de tendência de valores de coeficientes de uniformidade de distribuição maiores nos sistemas em que se utilizam gotejadores autocompensantes.

Como a função do sistema autocompesante é regular a pressão de entrada na linha lateral, para garantir a pressão de operação dos emissores dentro dos limites recomendados, a variação de vazão dos gotejadores se torna, ao longo da linha lateral, mínima, dentro do limite máximo de $10 \%$ da vazão de serviço do gotejador; contudo, mesmo nesses sistemas se encontraram valores de uniformidade abaixo dos recomendados visto que em alguns sistemas, como já referidos, há problemas com gotejadores que apresentavam a membrana de borracha rompida ou, ainda, que não a possuíam; já em sistemas em que se utilizam gotejadores não autocompensantes, consegue-se atingir altos valores de coeficiente de uniformidade (Figura 5B, desde que os sistemas sejam bem dimensionados e conduzidos, promovendo-se limpezas periódicas neles e reposição de emissores entupidos ou defeituosos. Salienta-se que, mesmo em um sistema autocompensante, são imprescindíveis a operação e a condução adequadas do sistema, pois isto é o que também irá diferenciar o desempenho correto do equipamento; além disso, a análise conjunta das Figuras $5 \mathrm{~A}$ e $\mathrm{B}$ justifica o grande número de sistemas com valores de CUD acima do recomendado, pois há maior número de sistemas novos, com menos de um ano de uso, sistemas autocompensantes e, ainda, sistemas que possuem as duas características, ideais para se obter valores acima do recomendado.

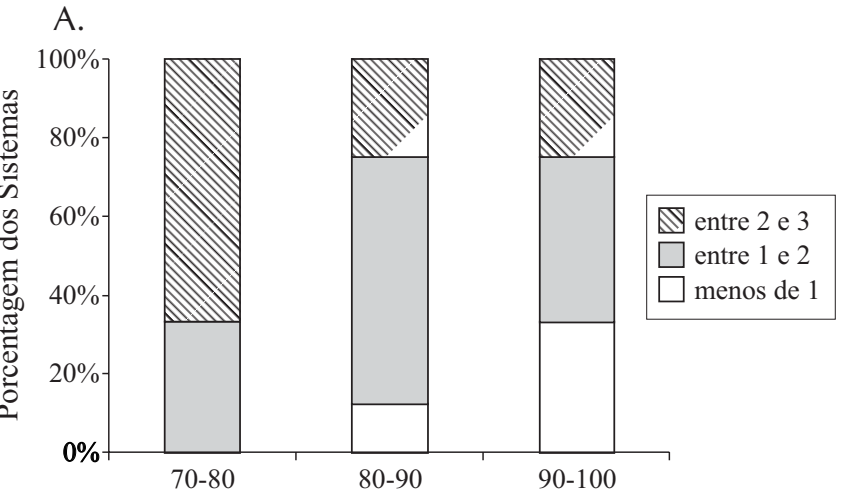

B.

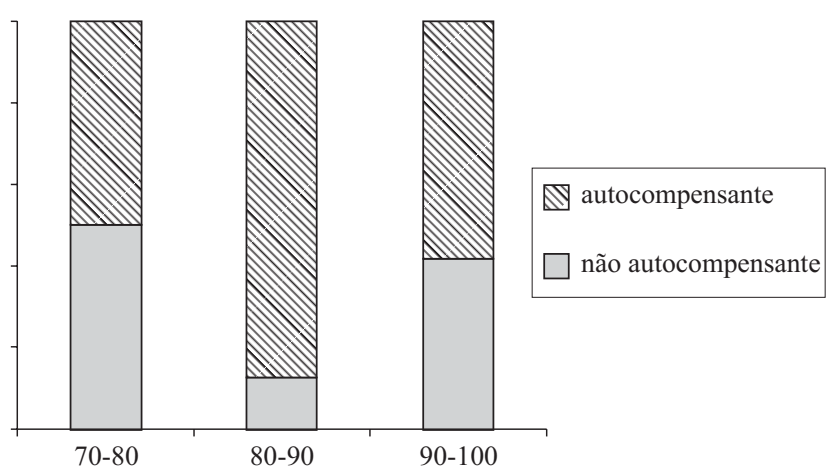

Coeficiente de Uniformidade de Distribuição (\%) 


\section{Porcentagem de área molhada}

$\mathrm{Na}$ Tabela 4 apresentam-se os valores de porcentagem de área molhada (PAM) dos sistemas avaliados nas regiões norte do Espírito Santo e cerrado de Minas Gerais, respectivamente, e nela se verifica-se, para a região norte do Espírito Santo, que os valores da PAM variaram de um mínimo de $19 \%$ a um máximo de $100 \%$, sendo este último valor obtido em um sistema alternativo, do tipo gravatinha, que se assemelha a uma microaspersão, em que toda a superfície do solo é molhada. Para a região de cerrado de Minas Gerais os valores da PAM variaram de um mínimo de $24 \%$ a um máximo também de $100 \%$; e este último valor foi obtido em um sistema cuja densidade de plantio era alta, acarretando molhamento total da superfície do solo.

Analisando-se a Tabela 4, em conjunto, observa-se que, dos 31 sistemas avaliados, apenas um obteve valor abaixo de $20 \%$, valor este recomendado por López et al. (1992) para regiões úmidas; entretanto, como usualmente o período de irrigação do café coincide com o período de baixa precipitação nas duas regiões estudadas, o valor mínimo de PAM recomendado seria de $33 \%$, caso em q ue o número de sistemas que não atingiram o valor mínimo recomendado é oito; além disso, em oito sistemas não foi possível se fazer a medição do bulbo molhado, visto que em dois deles choveu durante a avaliação, molhando a superfície do solo e, nos outros seis, o tempo gasto durante a avaliação foi inferior ao tempo normal de irrigação dos sistemas, levando à formação de um bulbo de tamanho inferior ao obtido em irrigações normais.

Ressalta-se que os irrigantes promovem as irrigações de maneira que o tempo de operação do sistema seja suficiente para que os bulbos molhados, formados na superfície do solo por gotejadores adjacentes, se interceptem; este fato pode ter

Tabela 4. Porcentagem de área molhada (PAM) para os sistemas avaliados na duas regiões

\begin{tabular}{|c|c|c|c|c|}
\hline \multirow{2}{*}{ Sistema } & \multicolumn{2}{|c|}{ Norte do Espírito Santo } & \multicolumn{2}{|c|}{ Cerrado de Minas Gerais } \\
\hline & Localização & PAM (\%) & Localização & PAM (\%) \\
\hline 1 & Nova Venécia & 19 & Patrocínio & 26 \\
\hline 2 & São Domingos do Norte & 35 & Coromandel & 30 \\
\hline 3 & Linhares & 36 & Araguari & 100 \\
\hline 4 & Rio Bananal & 100 & Romaria & - \\
\hline 5 & Rio Bananal & 28 & Rio Paranaíba & 32 \\
\hline 6 & São Roque do Canaã & 40 & Patrocínio & - \\
\hline 7 & Linhares & 31 & Araguari & 24 \\
\hline 8 & Linhares & 24 & Patrocínio & 65 \\
\hline 9 & Aracruz & 39 & Monte Carmelo & 24 \\
\hline 10 & Colatina & 38 & Monte Carmelo & 30 \\
\hline 11 & Jaguaré & 31 & Araguari & 25 \\
\hline 12 & Jaguaré & - & Presidente Olegário & - \\
\hline 13 & Linhares & 35 & Patos de Minas & 27 \\
\hline 14 & Sooretama & - & Patos de Minas & - \\
\hline 15 & Linhares & - & Patos de Minas & - \\
\hline \multirow[t]{2}{*}{16} & Linhares & 34 & & \\
\hline & Média & 37,7 & Média & 38,3 \\
\hline
\end{tabular}

contribuído para a obtenção dos valores mínimos recomendados para PAM porém, em contrapartida, o aumento do tempo de irrigação pode levar à aplicação de uma lâmina excessiva, que se perderá por percolação profunda.

\section{CONCLUSÕES}

1. Dos sistemas avaliados, $38,7 \%$ apresentaram valores de CUD acima de $90 \%$, classificados, portanto, como excelentes.

2. Os sistemas de irrigação, denominados alternativos, apresentaram valores de uniformidade abaixo do recomendado, principalmente por problemas relativos ao dimensionamento.

3. A presença de reguladores de pressão no início das linhas de derivação afetou, de maneira positiva, a uniformidade de distribuição de água nos sistemas avaliados.

4. Apenas um sistema apresentou valor de PAM abaixo do limite recomendado para regiões úmidas, que é de $20 \%$.

\section{LITERATURA CITADA}

Andrade Júnior, A. S.; Bastos, E. A.; Rodrigues, B. H. N. Efeito do tempo de uso na uniformidade de distribuição de água em um sistema de irrigação por gotejamento. Irriga, Botucatu, v.2, n.3, p.135-139, 1997.

Bonomo, R. Análise da irrigação na cafeicultura em áreas de cerrado de Minas Gerais. Viçosa: UFV, 1999. 224p. Tese Doutorado

Bralts, V. F.; Kesner, C. D. Drip irrigation field uniformity estimation. Transactions of the ASAE, St. Joseph, v.26, n.5, p.1369-1374, 1983.

Denículi, W.; Bernardo, S.; Thiábaut, J. T. L.; Sediyama, G. C. Uniformidade de distribuição de água, em condições de campo num sistema de irrigação por gotejamento. Revista Ceres, Viçosa, v.27, n.50, p.155-162, 1980.

Keller, J.; Bliesner, R. D. Sprinkle and trickle irrigation. New York: Avibook, 1990.649p.

Keller, J.; Karmeli D. Trickle irrigation design. Glendora: Rain Bird Sprinkler Manufacturing, 1975. 133p

López, R. J.; Abreu J. M. H.; Regalado, A. P.; Hernández, J. F. G. Riego localizado. Madrid: Mundi-Prensa, 1992. 405p.

Merriam, J. L.; Keller, J. Farm irrigation system evaluation: a guide for management. Logan: Utah State University, 1978. 271p.

Pizarro Cabello, F. Riegos localizados de alta frequencia:Goteo, microaspersion e exudacion. Madrid: Mundi-Prensa, 1990. 471p.

Sammis, T. W.; Wu, I. P. Effect of drip irrigation design and management on crop yield. Transactions of the ASAE, St. Joseph, v.28, n.4, p.832-837, 1985.

Solomon, K. H. Global uniformity of trickle irrigation systems. Transactions of the ASAE, St. Joseph, v.28, n.4, p.1151$1158,1985$. 